\title{
Treatment of Burn Scars with the Pinhole 4.0 Method Using a 10,600-nm Carbon Dioxide Laser
}

\author{
Sang Ju Lee ${ }^{1 *}$ \\ Joon Hyuk Suh2* \\ Jin Moon Kang ${ }^{1}$ \\ Young Koo Kim ${ }^{1}$ \\ Won Soon Chung ${ }^{1}$ \\ Beom Joon Kim² \\ Kui Young Park ${ }^{2}$
}

${ }^{1}$ Yonsei Star Skin \& Laser Clinic, Seoul, Korea

${ }^{2}$ Department of Dermatology, Chung-Ang

University College of Medicine, Seoul, Korea
Received November 8, 2015

Revised December 8, 2015

Accepted December 9, 2015

\section{Correspondence}

Kui Young Park

Department of Dermatology, Chung-Ang

University Hospital, 224-1 Heukseok-dong,

Dongjak-gu, Seoul 06973, Korea

Tel: +82-2-6299-1525

Fax: +82-2-823-1049

E-mail: momo920ahanmail.net

*They contributed this work equally as first authors.

(C) Korean Society for Laser Medicine and Surgery

(c) This is an open access article distributed under the terms of the Creative Commons Attribution NonCommercial License (http://creativecommons.org/ licenses/by-nc/4.0) which permits unrestricted noncommercial use, distribution, and reproduction in any medium, provided the original work is properly cited.

\begin{abstract}
Background and Objectives
Treatment of burn scars can be challenging due to their variable shape, color, and thickness. Several treatment modalities for burn scars have been introduced, but an ideal procedure has not yet been found. The pinhole method is a laser modality demonstrated as an effective treatment for burn scars. The aim of this study was to evaluate the efficacy and safety of the treatment of burn scars with a pinhole 4.0 method using a $10,600-\mathrm{nm}$ carbon dioxide $\left(\mathrm{CO}_{2}\right)$ laser.
\end{abstract}

\section{Materials and Methods}

We conducted a retrospective chart review of patients with hypertrophic burn scars treated using the pinhole 4.0 method.

\section{Results}

Eleven Korean patients with burn scars (mean age 38.0 years; range, 19-63 years) were treated using the pinhole 4.0 method. Subjects were treated via the pinhole method in single sessions. Two blinded dermatologists evaluated photographs taken at baseline and three months after treatment and assessed improvement using a quartile grading scale. Compared with baseline, all patients showed mild to moderate improvement (mean score: 3.0). The patient satisfaction questionnaire indicated a mean improvement score of 3.0.

\section{Conclusion}

The pinhole 4.0 method using a $\mathrm{CO}_{2}$ laser may be a safe and effective treatment option in patients with refractory hypertrophic burn scars.

\section{Key words}

Hypertrophic burn scar; Pinhole method; $\mathrm{CO}_{2}$ laser 


\section{INTRODUCTION}

Patients with burn scars frequently have lasting cosmetic and functional problems due to their scars. These are challenging lesions to treat and can result in emotional problems for patients, including decreased selfesteem, poor body image, and social withdrawal. ${ }^{12}$

Burn scars can be very challenging to treat due to their variable shape, color, and thickness. Several treatment modalities for burn scars have been introduced. These include medical approaches, such as silicone gel and intralesional corticosteroids injections, as well as surgical procedures, such as excision and skin graft. Recently, laser treatment modalities have emerged as a potentially effective arm of the treatment algorithm..$^{3-5}$ One of the most widely-used laser techniques employs fractional lasers in the pinhole method. The fractional laser, a relatively new modality, is able to improve texture and appearance of scars by penetrating the deep dermis. ${ }^{6}$ The pinhole method is a $\mathrm{CO}_{2}$ laser treatment technique that is effective for various skin lesions and could also be an option for scar treatment. Indeed, we previously reported mild to moderate improvement of scar appearance with the pinhole method. ${ }^{7}$ The pinhole 4.0 method combines usage of pinhole method with $\mathrm{CO}_{2}$ lasers and ablative fractional lasers.

In this study, we retrospectively reviewed our clinical experience and evaluated the efficacy and safety of the treatment of hypertrophic burn scars with the pinhole 4.0 method.

\section{MATERIALS AND METHODS}

\section{Patients}

We conducted a retrospective review of pinhole treatments performed in patients with hypertrophic burn scars. Informed consent was obtained from all patients, and the study was approved by our hospital's medical ethics committee. The study protocol conformed to the guidelines of the 1975 Declaration of Helsinki.

\section{Treatment}

A total of 11 Korean patients who underwent treatment with the pinhole method at our clinic were included in this study. Patient clinical characteristics are shown in Table 1. All patients were treated with a single session of the pinhole 4.0 method using 10,600-nm CO lasers (Ultrapulse $^{\circledR}$ Encore Laser; Lumenis, Inc., Santa Clara, CA, USA and eCO2 ${ }^{\circledR}$ Laser; Lutronic, Inc., Goyang, South Koreal. A topical eutectic mixture of $2.5 \%$ lidocaine and $2.5 \%$ prolicaine (EMLA cream, AstraZeneca AB, StraZenec, Sweden) was applied as local anesthetic one hour prior to the procedure. The pinhole 4.0 method is a concurrent treatment with two different devices in which two different modes were applied in one of them; the $\mathrm{CO}_{2}$ Laser with parameter of $100 \mathrm{~mJ}$ and $75 \mathrm{spots} / \mathrm{cm}^{2}$ density and Ultrapulse Encore Laser with parameter of $100 \mathrm{~mJ}$ and $1 \%$ density in ScaarFx mode and 3.0 W in Single mode were used to make multiple small holes. Holes were approximately 0.12 to $2 \mathrm{~mm}$ in diameter and spaced $3 \mathrm{~mm}$ apart over the scar lesion.

Table 1. Characteristics of patients with hypertrophic burn scars

\begin{tabular}{|c|c|c|c|c|c|}
\hline \multirow{2}{*}{ Patients } & \multirow{2}{*}{ Sex/Age } & \multirow{2}{*}{ Location } & \multicolumn{2}{|c|}{ Clinical improvement } & \multirow{2}{*}{ Adverse events ${ }^{\ddagger}$} \\
\hline & & & Improvement* & Patient satisfaction $^{\dagger}$ & \\
\hline 1 & $F / 39$ & Arm & 3 & 3 & $\mathrm{~N}$ \\
\hline 2 & $F / 32$ & Wrist & 4 & 4 & $\mathrm{~N}$ \\
\hline 3 & $F / 47$ & Buttock & 3 & 3 & $\mathrm{~N}$ \\
\hline 4 & $F / 39$ & Face & 3 & 2 & $\mathrm{~N}$ \\
\hline 5 & $M / 33$ & Neck & 4 & 4 & $\mathrm{~N}$ \\
\hline 6 & $F / 22$ & Leg & 3 & 4 & $\mathrm{~N}$ \\
\hline 7 & $\mathrm{~F} / 36$ & Leg & 2 & 2 & $\mathrm{~N}$ \\
\hline 8 & $F / 19$ & Face & 1 & 1 & $\mathrm{~N}$ \\
\hline 9 & $F / 38$ & Back & 3 & 3 & $\mathrm{~N}$ \\
\hline 10 & $\mathrm{~F} / 63$ & Arm & 4 & 4 & $\mathrm{~N}$ \\
\hline 11 & $\mathrm{~F} / 50$ & Buttock & 3 & 3 & $\mathrm{~N}$ \\
\hline
\end{tabular}

\footnotetext{
* Improvement was evaluated using a quartile grading scale; grade 1 , less than $25 \%=$ minimal to no improvement; grade $2,26 \%$ to $50 \%=$ moderate improvement; grade $3,51 \%$ to $75 \%$ = marked improvement; and grade 4 , more than $75 \%=$ near total improvement.

${ }^{\dagger}$ Patients were surveyed three months after treatment about their overall level of satisfaction as follows: 4-very satisfied, 3-satisfied, 2-slightly satisfied, and 1-unsatisfied.

${ }^{\ddagger} \mathrm{N}$, none.
} 

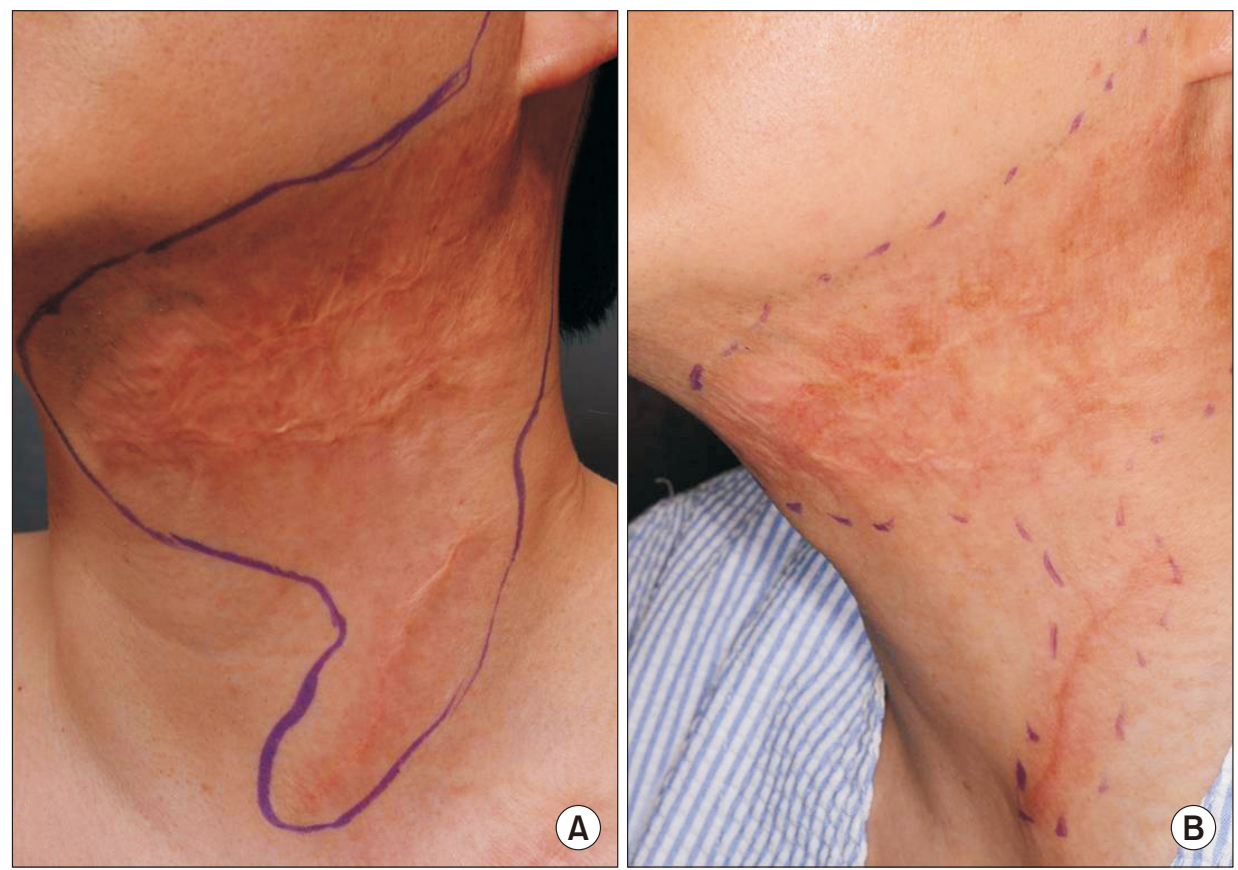

Fig. 1. Photographs of patient 5 (A) before and (B) three months after one pinhole 4.0 treatment session.

\section{Evaluation}

A clinical photograph of the hypertrophic burn scar was taken with the same camera at every visit. Clinical photographs of the scars before and three months after treatmentwere assessed using side-by-side comparison and objectively assessed by two independent dermatologists for all patients. Patients and dermatologists evaluated the efficacy of improvement using a quartile grading scale (Grade 1, <25\% improvement; Grade 2, 25-50\% improvement; Grade3, 51-75\% improvement; and Grade 4, >75\% improvement).

\section{RESULTS}

All eleven patients in this study were Korean. One patient was male and ten were female. The age at presentation ranged from 19 to 63 years (mean age, 38.0 years). Lesions were on the face, neck, arm, wrist, hands, back, and buttock. Two independent dermatologists' comparisons of the baseline and three months after treatment photographs showed mild to moderate improvement for all patients (Grades 1-4; mean score: 3.0). Three patients (27\%) showed Grade 4 improvement; six (55\%) Grade 3; one (9\%) Grade 2 improvement; and one (9\%) Grade 1 improvement. The patient satisfaction questionnaire three months after treatment revealed a mean improvement score of 3.0. Four patients reported a satisfaction rating of Grade 4; four, Grade 3; two, Grade 2; and one, Grade 1 (Table 1).
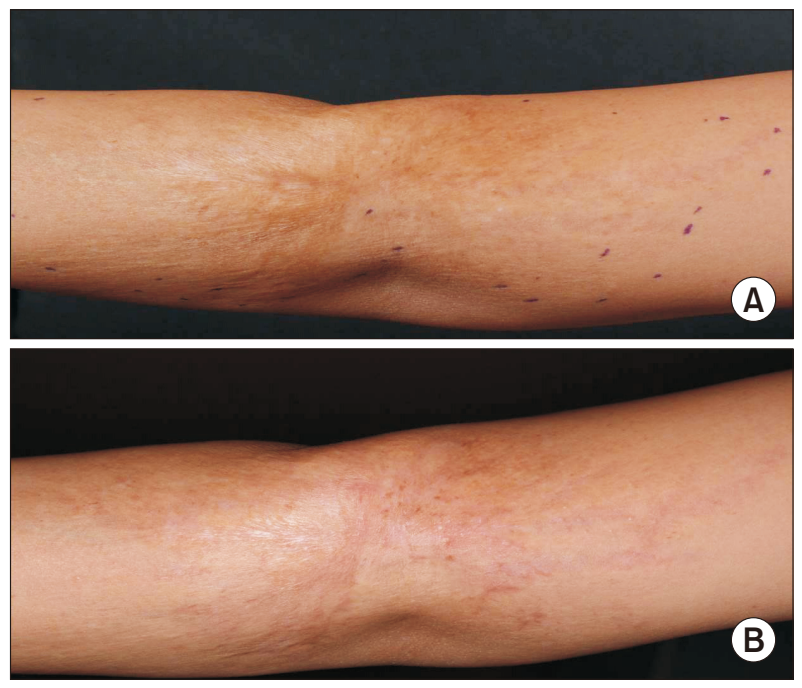

Fig. 2. Photographs of patient 10 (A) before and (B) threemonths after one pinhole treatment session.

All patients tolerated laser treatment well, and in some patients, excellent improvement of the appearance of hypertrophic burn scars was achieved. There were no complications such as permanent pigment change, ulceration, or infection during the clinical study.

\section{DISCUSSION}

Hypertrophic scarsare a fibrotic pathology arising from the fibroproliferation that occurs after damage to the deep 
dermis by burns or trauma. ${ }^{8}$ Hypertrophic burn scars may induce, or are frequently associated with, erythema with contractures, itching, and pain. Several different modalities have been developed to improve hypertrophic burn scars, including medical procedures such as pressure therapy, silicone gel, introlesional corticosteroid injections, surgical procedures, and laser therapy. ${ }^{3,4,5}$ However, there are limitations to using these treatments alone.

Because of these limitations, combination therapy has been attempted by several groups. For example, Cho et al. ${ }^{9}$ showed that the combination of pinhole and microneedle-induced collagen induction therapy result in a greater improvement in the scar than either method alone. Similarly, Lee et al. ${ }^{7}$ suggested that combination therapy with laser-cision and a pinhole method using a $\mathrm{CO}_{2}$ laser is effective for improving hypertrophic burn scars.

The pinhole method is an attractive option for treating burn scars. This method involves making multiple small holes that penetrate from the epidermis to the deeper dermis using a $\mathrm{CO}_{2}$ laser. This induces breakdown of irregular and thick collagen bundles resulting from thermal damage, as well as regeneration and realignment of collagen bundles at the microscopic level. Clinically, this manifests as improves texture and thickness of the scar tissue. ${ }^{10}$ However, the existing pinhole method requires several sessions to achieve satisfactory results.

In this study, we investigated the efficacy of a combination therapy-the pinhole 4.0 method-for hypertrophic burn scars using a $\mathrm{CO}_{2}$ laser and ablative fractional laser. The method is a concurrent technique with three different modes: eCO2, ScaarFx, and CO2 modes. The novel pinhole 4.0 method demonstrated clear improvement with just one session by affecting the fundamental cause of the scar with these various lasers. In particular, the ScaarFxmode is able to penetrate up to $4 \mathrm{~mm}$ beneath the skin, which allows for effective treatment of thick scars that cannot be treated with other lasers."

This study is limited by a small sample size, a short follow-up period, and its retrospective design. Therefore, additional larger, prospective, randomized-controlled trials will be needed to determine whether a single session of the pinhole 4.0 method is more effective than several sessions of previous pinhole method.

In this study, we report that the efficient pinhole 4.0 method can be used for burn scar treatment and that it shows no severe complications other than temporary erythema or post-inflammatory hyperpigmentation. We suggest that the pinhole 4.0 method using a $\mathrm{CO}_{2}$ laser may be a safe and effective treatment option for patients with refractory hypertrophic burn scars.

\section{REFERENCES}

1. Rumsey N, Clarke A, White P. Exploring the psychosocial concerns of outpatients with disfiguring conditions. J Wound Care 2003;12:247-52.

2. Leblebici B, Adam M, Bağiş S, Tarim AM, Noyan T, Akman $M N$, et al. Quality of life after burn injury: the impact of joint contracture. J Burn Care Res 2006;27:864-8.

3. Motamed S, Hasanpoor SE, Moosavizadeh SM, Arasteh E. Treatment of flexion contractures following burns in extremities. Burns 2006;32:1017-21.

4. Hultman CS, Teotia S, Calvert C, Thornton S, Schram J. STARplasty for reconstruction of the burned web space: introduction of an alternative technique for the correction of dorsal neosyndactyly. Ann Plast Surg 2005;54:281-7.

5. Hultman CS, Edkins RE, Lee CN, Calvert CT, Cairns BA. Shine on: review of laser- and light-based therapies for the treatment of burn scars. Dermatol Res Pract 2012;2012:243651.

6. Waibel J, Wulkan AJ, Lupo M, Beer K, Anderson RR. Treatment of burn scars with the 1,550 nm nonablative fractional Erbium Laser. Lasers Surg Med 2012;44:441-6.

7. Lee SJ, Yeo IK, Kang JM, Chung WS, Kim YK, Kim BJ, et al. Treatment of hypertrophic burn scars by combination lasercision and pinhole method using a carbon dioxide laser. Lasers Surg Med 2014;46:380-4.

8. Yang QQ, Yang SS, Tan JL, Luo GX, He WF, Wu J. Process of hypertrophic scar formation: expression of eukaryotic initiation factor 6. Chin Med J (Engl) 2015;128:2787-91.

9. Cho SB, Lee SJ, Kang JM, Kim YK, Kim TY, Kim DH. The treatment of burn scar-induced contracture with the pinhole method and collagen induction therapy: a case report. J Eur Acad Dermatol Venereol 2008;22:513-4.

10. Whang SW, Lee KY, Cho SB, Lee SJ, Kang JM, Kim YK, et al. Burn scars treated by pinhole method using a carbon dioxide laser. J Dermatol 2006;33:869-72.

11. El-Zawahry BM, Sobhi RM, Bassiouny DA, Tabak SA. Ablative CO2 fractional resurfacing in treatment of thermal burn scars: an open-label controlled clinical and histopathological study. J Cosmet Dermatol. In press 2015. 